\title{
A BATCH-ARRIVAL QUEUE WITH MULTIPLE SERVERS AND FUZZY PARAMETERS: PARAMETRIC PROGRAMMING APPROACH
}

\author{
Jau-Chuan $\mathrm{Ke}^{*}$, Hsin-I Huang ${ }^{* *}$, Chuen-Horng Lin ${ }^{* *}$ \\ *Department of Statistics, National Taichung Institute of Technology, Taichung 404, \\ Taiwan, R.O.C. jauchuan@ntit.edu.tw; \\ **Department of Information Management, National Taichung Institute of \\ Technology, Taichung 404, Taiwan, R.O.C. syhuang@ntit.edu.tw; linch@ntit.edu.tw
}

\begin{abstract}
This work constructs the membership functions of the system characteristics of a batch-arrival queuing system with multiple servers, in which the batch-arrival rate and customer service rate are all fuzzy numbers. The $\alpha$-cut approach is used to transform a fuzzy queue into a family of conventional crisp queues in this context. By means of the membership functions of the system characteristics, a set of parametric nonlinear programs is developed to describe the family of crisp batch-arrival queues with multiple servers. A numerical example is solved successfully to illustrate the validity of the proposed approach. Because the system characteristics are expressed and governed by the membership functions, the fuzzy batch-arrival queues with multiple servers are represented more accurately and the analytic results are more useful for system designers and practitioners.
\end{abstract}

Keywords- Fuzzy sets, Membership function, Multiple server, Nonlinear programming

\section{INTRODUCTION}

Queueing models with multiple servers are effective methods for performance analysis of computer and telecommunication systems, manufacturing/production systems and inventory control (Kleinrock [11], Buzacott and Shanthikumar [1], Gross and Harris [7], Trivedi [19]). In general, these analyses consider a queueing system where requests for service arrive in units, one at a time (single-unit arrival). In many practical situations, however, requests for service usually arrive in batches. For example, in manufacturing systems of the job-shop type, each job order often requires the manufacture of more than one unit; in computer communication systems, messages which are to be transmitted could consist of a random number of packets. If the usual crisp batch-arrival queues with multiple servers can be extended to fuzzy batch-arrival queues, such queueing models would have wider applications.

For queueing models with multiple servers under various considerations, the $\mathrm{M} / \mathrm{M} / \mathrm{c}$ vacation systems with a single-unit arrival have attracted much attention from numerous researchers since Levy and Yechiali [12]. The extensions of this model can be referred to Vinod [20], Igaki [8], Tian et al. [17], Tian and Xu [18], and Zhang and Tian [23, 24]. Zhang and Tian $[23,24]$ studied the $\mathrm{M} / \mathrm{M} / \mathrm{c}$ vacation systems with a single-unit arrival and a "partial server vacation policy". They proved several conditional stochastic decomposition results for the queue length and waiting time. Chao and Zhao [3] investigated the $\mathrm{GI} / \mathrm{M} / \mathrm{c}$ vacation models with a single-unit arrival and provided iterative algorithms for computing the stationary probability distributions.

In the literature described above, customer inter-arrival times and customer service times are required to follow certain probability distributions with fixed parameters. 
However, in many real-world applications, the parameter distributions may only be characterized subjectively; that is, the arrival and service are typically described in everyday language summaries of central tendency, such as "the mean arrival rate is around 5 per day", or "the mean service rate is about 10 per hour", rather than with complete probability distributions. In other words, these system parameters are both possibilistic and probabilistic. Thus, fuzzy queues are potentially much more useful and realistic than the commonly used crisp queues (see Li and Lee [13] and Zadeh [22]). By extending the usual crisp batch-arrival queues to fuzzy batch-arrival queues in the context of multiple servers, these queuing models become appropriate for a wider range of applications.

$\mathrm{Li}$ and Lee [13] investigated the analytical results for two typical fuzzy queues (denoted $M / F / 1 / \infty$ and $F M / F M / 1 / \infty$, where $F$ represents fuzzy time and $F M$ represents fuzzified exponential distributions) using a general approach based on Zadeh's extension principle (see also Prade [15] and Yager [21]), the possibility concept and fuzzy Markov chains (see Stanford [16]). A useful modeling and inferential technique would be applied their approach to general fuzzy queuing problems (see Stanford [16]). However, their approach is complicated and not suitable for computational purposes; moreover, it cannot easily be used to derive analytic results for other complicated queuing systems (see Negi and Lee [14]). In particular, it is very difficult to apply this approach to fuzzy queues with more fuzzy variables or multiple servers. Negi and Lee [14] proposed a procedure using $\alpha$-cuts and two-variable simulation to analyze fuzzy queues (see also Chanas and Nowakowski [2]). Unfortunately, their approach provides only crisp solutions; i.e., it does not fully describe the membership functions of the system characteristics. Using parametric programming, Kao et al. [9] constructed the membership functions of the system characteristics for fuzzy queues and successfully applied them to four simple fuzzy queue models: $M / F / 1 / \infty, F / M / 1 / \infty, F / F / 1 / \infty$ and $F M / F M / 1 / \infty$. Recently, Chen [4,5] developed $F M / F M / 1 / L$ and $F M / F M^{[K] / 1 / \infty}$ fuzzy systems using the same approach.

All previous researches on fuzzy queuing models are focused on ordinary queues with a single server. In this paper, we develop an approach that provides system characteristics for batch-arrival queues with multiple servers and fuzzy parameters: fuzzified exponential batch-arrival and service rates. Through $\alpha$-cuts and Zadeh's extension principle, we transform the fuzzy queues to a family of crisp queues. As $\alpha$ varies, the family of crisp queues is described and solved using parametric nonlinear programming (NLP). The NLP solutions completely and successfully yield the membership functions of the system characteristics, including the expected number of customers in the system and the expected waiting time in the queue.

The remainder of this paper is organized as follows. Section 2 presents the system characteristics of standard and fuzzy batch-arrival queuing models with multiple servers. In Section 3, a mathematical programming approach is developed to derive the membership functions of these system characteristics. To demonstrate the validity of the proposed approach, one realistic numerical example is described and solved. Discussion is provided in Section 4, and conclusions are drawn in Section 5. For notational convenience, our model in this paper is hereafter denoted $F M^{[x]} / F M / \mathrm{c}$. 


\section{FUZZY BATCH QUEUE WITH MULTIPLE SERVERS}

We consider a batch-arrival queuing system with $\mathrm{c}$ servers where the customers arrive in batches to occur according to a compound Poisson process with batch-arrival rate $\lambda$. Let $A_{k}$ denote the number of customers belonging to the $k^{\text {th }}$ arrival batch, where $A_{k}, k=1,2,3, \Lambda$, are with a common distribution $\operatorname{Pr}\left[A_{k}=n\right]=a_{n}, n=1,2,3, \Lambda$, and $E[A]=\sum_{n=1}^{\infty} n a_{n}$. Customers arriving at the service facility (servers) form a single-file queue and are served in order. The service time for each of all c servers is exponentially distributed with rate $\mu$ and each server can serve only one customer at a time. Customers who upon entry the service facility find that all servers are busy have to wait in the queue until any one server is available. Let $N_{s}$ and $W_{q}$ represents the expected number of customers in the system and the expected waiting time in the queue, respectively. Through a Markov process, we can easily obtain $N_{s}$ and $W_{q}$ in terms of system parameters

$$
\begin{aligned}
N_{s} & =\frac{\lambda(2 E[A]+E[A(A-1)])+2 \mu \cdot \sum_{n=0}^{c-1} n(c-n) P_{n}(\lambda, \mu)}{2(c \mu-\lambda E[A])}, \\
W_{q} & =\frac{\lambda(2 E[A]+E[A(A-1)])+2 \mu \cdot \sum_{n=0}^{c-1} n(c-n) P_{n}(\lambda, \mu)}{2 \lambda E[A](c \mu-\lambda E[A])}-\frac{1}{\mu},
\end{aligned}
$$

where $P_{n}(\lambda, \mu)$ represents the probability that there are $n$ customers in the system. And the probability depends on $\lambda$ and $\mu$. In steady-state, it is necessary that we have $0<\frac{\lambda E[A]}{c \mu}<1$.

To extend the applicability of the batch-arrival queuing model with multiple servers, we allow for fuzzy specification of system parameters. Suppose the batch-arrival rate $\lambda$ for customers and service rate $\mu$ for each server are approximately known and can be represented by the fuzzy sets $\tilde{\lambda}$ and $\tilde{\mu}$. Let $\phi_{\tilde{\lambda}}(x)$ and $\phi_{\widetilde{\mu}}(y)$ denote the membership functions of $\tilde{\lambda}$ and $\tilde{\mu}$. We then have the following fuzzy sets:

$$
\begin{aligned}
& \tilde{\lambda}=\left\{\left(x, \phi_{\widetilde{\lambda}}(x)\right) \mid x \in X\right\}, \\
& \tilde{\mu}=\left\{\left(y, \phi_{\widetilde{\mu}}(y)\right) \mid y \in Y\right\},
\end{aligned}
$$

where $X$ and $Y$ are the crisp universal sets of the batch-arrival and service rates.

Let $f(x, y)$ denote the system characteristic of interest. Since $\tilde{\lambda}$ and $\tilde{\mu}$ are fuzzy numbers, $f(\tilde{\lambda}, \tilde{\mu})$ is also a fuzzy number. Following Zadeh's extension principle (see Yager [21] and Zadeh [22]), the membership function of the system characteristic $f(\tilde{\lambda}, \tilde{\mu})$ is defined as:

$$
\phi_{f(\tilde{\lambda}, \widetilde{\mu})}(z)=\sup _{x \in X, y \in Y, 0<x E[A] / c y<1} \min \left\{\phi_{\widetilde{\lambda}}(x), \phi_{\widetilde{\mu}}(y) \mid z=f(x, y)\right\},
$$

Assume that the system characteristic of interest is the expected number of 
customers in the system. It follows from (1) that the expected number of customers in the system is:

$$
f(x, y)=\frac{x(2 E[A]+E[A(A-1)])+2 y \cdot \sum_{n=0}^{c-1} n(c-n) P_{n}(x, y)}{2(c y-x E[A])} .
$$

The membership function for the expected number of customers in the system is:

$\phi_{\widetilde{N}_{s}}(z)=\sup _{x \in X, y \in Y, 0<x E[A] / c y<1} \min \left\{\phi_{\widetilde{\lambda}}(x), \phi_{\widetilde{\mu}}(y) \mid z=\frac{x(2 E[A]+E[A(A-1)])+2 y \cdot \sum_{n=0}^{c-1} n(c-n) P_{n}(x, y)}{2(c y-x E[A])}\right\}$.

Unfortunately, the membership function is not expressed in the usual form, making it very difficult to imagine its shape. In this paper we approach the representation problem using a mathematical programming technique. Parametric NLPs are developed to find the $\alpha$-cuts of $f(\tilde{\lambda}, \tilde{\mu})$ based on the extension principle.

\section{PARAMETRIC NONLINEAR PROGRAMMING}

To re-express the membership function $\phi_{\widetilde{N}_{s}}(z)$ of $\tilde{N}_{s}$ in an understandable and usable form, we adopt Zadeh's approach, which relies on $\alpha$-cuts of $\widetilde{N}_{s}$. Definitions for the $\alpha$-cuts of $\tilde{\lambda}$ and $\tilde{\mu}$ as crisp intervals are as follows:

$$
\begin{aligned}
& \lambda(\alpha)=\left[x_{\alpha}^{L}, \mathrm{x}_{\alpha}^{U}\right]=\left[\min _{x \in X}\left\{x \mid \phi_{\widetilde{\lambda}}(x) \geq \alpha\right\}, \max _{x \in X}\left\{x \mid \phi_{\widetilde{\lambda}}(x) \geq \alpha\right\}\right], \\
& \mu(\alpha)=\left[y_{\alpha}^{L}, \mathrm{y}_{\alpha}^{U}\right]=\left[\min _{y \in Y}\left\{y \mid \phi_{\widetilde{\mu}}(y) \geq \alpha\right\}, \max _{y \in Y}\left\{y \mid \phi_{\widetilde{\mu}}(y) \geq \alpha\right\}\right],
\end{aligned}
$$

The constant batch-arrival and service rates are shown as intervals when the membership functions are no less than a given possibility level for $\alpha$. As a result, the bounds of these intervals can be described as functions of $\alpha$ and can be obtained as: $x_{\alpha}^{L}=\min \phi_{\tilde{\lambda}}^{-1}(\alpha), \quad x_{\alpha}^{U}=\max \phi_{\tilde{\lambda}}^{-1}(\alpha), \quad y_{\alpha}^{L}=\min \phi_{\widetilde{\mu}}^{-1}(\alpha) \quad, \quad$ and $\quad y_{\alpha}^{U}=\max \phi_{\widetilde{\mu}}^{-1}(\alpha)$. Therefore, we can use the $\alpha$-cuts of $\tilde{N}_{s}$ to construct its membership function since the membership function defined in (6) is parameterized by $\alpha$.

Using Zadeh's extension principle, $\phi_{\widetilde{N}_{s}}(z)$ is the minimum of $\phi_{\widetilde{\lambda}}(x)$ and $\phi_{\widetilde{\mu}}(y)$. To derive the membership function $\phi_{\widetilde{N}_{s}}(z)$, we need at least one of the following cases to hold such that $\mathrm{z}=\frac{x(2 E[A]+E[A(A-1)])+2 y \cdot \sum_{n=0}^{c-1} n(c-n) P_{n}(x, y)}{2(c y-x E[A])}$ satisfies $\phi_{\widetilde{N}_{s}}(z)=\alpha$ :

$$
\begin{aligned}
& \text { Case (i): }\left(\phi_{\widetilde{\lambda}}(x)=\alpha, \phi_{\widetilde{\mu}}(y) \geq \alpha\right), \\
& \text { Case (ii): }\left(\phi_{\widetilde{\lambda}}(x) \geq \alpha, \phi_{\widetilde{\mu}}(y)=\alpha\right),
\end{aligned}
$$

This can be accomplished using parametric NLP techniques. The NLP to find the lower and upper bounds of the $\alpha$-cut of $\phi_{\widetilde{N}_{s}}(z)$ for Case (i) are: 


$$
\begin{aligned}
\left(N_{s}\right)_{\alpha}^{L_{1}}=\min \frac{x(2 E[A]+E[A(A-1)])+2 y \cdot \sum_{n=0}^{c-1} n(c-n) P_{n}(x, y)}{2(c y-x E[A])}, \\
\left(N_{s}\right)_{\alpha}^{U_{1}}=\max \frac{x(2 E[A]+E[A(A-1)])+2 y \cdot \sum_{n=0}^{c-1} n(c-n) P_{n}(x, y)}{2(c y-x E[A])},
\end{aligned}
$$

and for Case (ii) are:

$$
\begin{aligned}
& \left(N_{s}\right)_{\alpha}^{L_{2}}=\min \frac{x(2 E[A]+E[A(A-1)])+2 y \cdot \sum_{n=0}^{c-1} n(c-n) P_{n}(x, y)}{2(c y-x E[A])}, \\
& \left(N_{s}\right)_{\alpha}^{U_{2}}=\max \frac{x(2 E[A]+E[A(A-1)])+2 y \cdot \sum_{n=0}^{c-1} n(c-n) P_{n}(x, y)}{2(c y-x E[A])} .
\end{aligned}
$$

From the definitions of $\lambda(\alpha)$ and $\mu(\alpha)$ in (7), $x \in \lambda(\alpha)$ and $y \in \mu(\alpha)$ can be replaced by $x \in\left[x_{\alpha}^{L}, x_{\alpha}^{U}\right]$ and $y \in\left[y_{\alpha}^{L}, y_{\alpha}^{U}\right]$. The $\alpha$-cuts form a nested structure with respect to $\alpha$ (see Kaufmann [10] and Zimmermann [25]); i.e., given $0<\alpha_{2}<\alpha_{1} \leq 1$, we have $\left[x_{\alpha_{1}}^{L}, x_{\alpha_{1}}^{U}\right] \subseteq\left[x_{\alpha_{2}}^{L}, x_{\alpha_{2}}^{U}\right]$ and $\left[y_{\alpha_{1}}^{L}, y_{\alpha_{1}}^{U}\right] \subseteq\left[y_{\alpha_{2}}^{L}, y_{\alpha_{2}}^{U}\right]$. Therefore, (8a) and (8c) have the same smallest element and (8b) and (8d) have the same largest element. To find the membership function $\phi_{\widetilde{N}_{s}}(z)$, it suffices to find the left and right shape functions of $\phi_{\widetilde{N}_{s}}(z)$, which is equivalent to finding the lower bound $\left(N_{s}\right)_{\alpha}^{L}$ and upper bound $\left(N_{s}\right)_{\alpha}^{U}$ of the $\alpha$-cuts of $\tilde{N}_{s}$, which can be rewritten as:

$$
\begin{aligned}
\left(N_{s}\right)_{\alpha}^{L}=\min \frac{x(2 E[A]+E[A(A-1)])+2 y \cdot \sum_{n=0}^{c-1} n(c-n) P_{n}(x, y)}{2(c y-x E[A])} \\
\text { s.t. } x_{\alpha}^{L} \leq x \leq x_{\alpha}^{U} \text { and } y_{\alpha}^{L} \leq y \leq y_{\alpha}^{U}, \\
\left(N_{s}\right)_{\alpha}^{U}=\max \frac{x(2 E[A]+E[A(A-1)])+2 y \cdot \sum_{n=0}^{c-1} n(c-n) P_{n}(x, y)}{2(c y-x E[A])} \\
\text { s.t. } x_{\alpha}^{L} \leq x \leq x_{\alpha}^{U} \text { and } y_{\alpha}^{L} \leq y \leq y_{\alpha}^{U},
\end{aligned}
$$

At least one of $x$ and $y$ must hit the boundaries of their $\alpha$-cuts to satisfy $\phi_{\widetilde{N}_{s}}(z)=\alpha$. This model is a set of mathematical programs with boundary constraints and lends itself to the systematic study of how the optimal solutions change with $x_{\alpha}^{L}$, $x_{\alpha}^{U}, y_{\alpha}^{L}$, and $y_{\alpha}^{U}$ as $\alpha$ varies over $(0,1]$. The model is a special case of parametric NLPs (see Gal [6]).

The crisp interval $\left[\left(N_{s}\right)_{\alpha}^{L},\left(N_{s}\right)_{\alpha}^{U}\right]$ obtained from (9) represents the $\alpha$-cuts of $\tilde{N}_{s}$. Again, by applying the results of Kaufmann [10] and Zimmermann [25] and convexity properties to $\widetilde{N}_{s}$, we have $\left(N_{s}\right)_{\alpha_{1}}^{L} \geq\left(N_{s}\right)_{\alpha_{2}}^{L}$ and $\left(N_{s}\right)_{\alpha_{1}}^{U} \leq\left(N_{s}\right)_{\alpha_{2}}^{U}$, where $0<\alpha_{2}<\alpha_{1} \leq 1$. In other words, $\left(N_{s}\right)_{\alpha}^{L}$ increases and $\left(N_{s}\right)_{\alpha}^{U}$ decreases as $\alpha$ 
increases. Consequently, the membership function $\phi_{\widetilde{N}_{s}}(z)$ can be found from (9).

If both $\left(N_{s}\right)_{\alpha}^{L}$ and $\left(N_{s}\right)_{\alpha}^{U}$ in (9) are invertible with respect to $\alpha$, then a left shape function $L(z)=\left[\left(N_{s}\right)_{\alpha}^{L}\right]^{-1}$ and a right shape function $R(z)=\left[\left(N_{s}\right)_{\alpha}^{U}\right]^{-1}$ can be derived, from which the membership function $\phi_{\widetilde{N}_{s}}(z)$ is constructed:

$$
\phi_{\widetilde{N}_{s}}(z)= \begin{cases}L(z), & \left(N_{\mathrm{s}}\right)_{\alpha=0}^{L} \leq z \leq\left(N_{\mathrm{s}}\right)_{\alpha=1}^{L}, \\ 1, & \left(N_{\mathrm{s}}\right)_{\alpha=1}^{L} \leq z \leq\left(N_{\mathrm{s}}\right)_{\alpha=1}^{U}, \\ R(z), & \left(N_{\mathrm{s}}\right)_{\alpha=1}^{U} \leq z \leq\left(N_{\mathrm{s}}\right)_{\alpha=0}^{U} .\end{cases}
$$

In most cases, the values of $\left(N_{s}\right)_{\alpha}^{L}$ and $\left(N_{s}\right)_{\alpha}^{U}$ cannot be solved analytically. Consequently, a closed-form membership function for $\phi_{\widetilde{N}_{s}}(z)$ cannot be obtained. However, the numerical solutions for $\left(N_{s}\right)_{\alpha}^{L}$ and $\left(N_{s}\right)_{\alpha}^{U}$ at different possibility levels can be collected to approximate the shapes of $L(z)$ and $R(z)$. That is, the set of intervals $\left\{\left[\left(N_{s}\right)_{\alpha}^{L},\left(N_{s}\right)_{\alpha}^{U}\right] \mid \alpha \in[0,1]\right\}$ shows the shape of $\phi_{\widetilde{N}_{s}}(z)$, although the exact function is not known explicitly.

Note that the membership functions for the expected waiting time in the queue can be expressed in a similar manner.

\section{NUMERICAL EXAMPLE}

This section we present one example motivated by real-life systems to demonstrate the practical use of the proposed approach, which is based on http://www.macaudata.com/macauweb/book175/html/19301.htm

Example: Considering one sewerage treatment system collects sewage from the urban areas and sends them to the sewerage treatment plant. The sewerage treatment plant has three supply pipes (referred to 3-servers). Each pipe can settle the larger solids and put the settled into the chemical process tank. After the chemical process, the treated water is discharged to the sea. We assume that the number of arriving sewage solids each time follows a geometric distribution with parameter $p=0.5$; i.e., the size of arriving sewage solids $A$ is $\operatorname{Pr}(A=k)=0.5(1-0.5)^{k-1}, k=1,2, \Lambda$. Clearly, this problem can be described by $\mathrm{FM}^{[\mathrm{x}]} / \mathrm{FM} / 3$ system. For efficiency, the management wants to get the system characteristics such as the expected number of sewage solids in the system and the expected waiting time in the queue.

Suppose the batch-arrival rate and service rate are trapezoidal fuzzy numbers represented by $\tilde{\lambda}=[1,2,3,4]$ and $\tilde{\mu}=[11,12,13,14]$. First, it is easy to find that $\left[x_{\alpha}^{L}, x_{\alpha}^{U}\right]=[1+\alpha, 4-\alpha]$ and $\left[y_{\alpha}^{L}, y_{\alpha}^{U}\right]=[11+\alpha, 14-\alpha]$. Next, it is obvious that when $x=x_{\alpha}^{U}$ and $y=y_{\alpha}^{L}$, the expected number of sewage solids in the system attains its maximum value, and when $x=x_{\alpha}^{L}$, and $y=y_{\alpha}^{U}$, the expected number of sewage solids in the system attains its minimum value. According to (9), the $\alpha$-cuts of $\tilde{N}_{s}$ are:

$$
\left(N_{s}\right)_{\alpha}^{L}=\frac{27200+23460 \alpha-3630 \alpha^{2}+110 \alpha^{3}}{99200-21000 \alpha+1275 \alpha^{2}-25 \alpha^{3}},
$$




$$
\left(N_{s}\right)_{\alpha}^{U}=\frac{67880-4650 \alpha-2640 \alpha^{2}-110 \alpha^{3}}{47000+14025 \alpha+1050 \alpha^{2}+25 \alpha^{3}} .
$$

With the help of MATLAB ${ }^{\circledR} 7.0 .4$, the membership function is:

where:

$$
\phi_{\widetilde{N}_{s}}(z)= \begin{cases}L(z), & \frac{17}{62} \leq z \leq \frac{4714}{7945} \\ 1, & \frac{4714}{7945} \leq z \leq \frac{112}{115} \\ R(z), & \frac{112}{115} \leq z \leq \frac{1697}{1175}\end{cases}
$$

$$
\begin{gathered}
L(z)=\frac{3(-1+\sqrt{3} i) P^{\frac{2}{3}}+2(85 z+242) P^{\frac{1}{3}}-3(1+\sqrt{3} i)\left(25 z^{2}+280 z+2684\right)}{2(5 z+22) P^{\frac{1}{3}}}, \\
R(z)=\frac{3 Q^{\frac{2}{3}}-2(35 z+88) Q^{\frac{1}{3}}+3\left(25 z^{2}+280 z+2684\right)}{(5 z+22) Q^{\frac{1}{3}}},
\end{gathered}
$$

with:

$$
\begin{aligned}
& P=-1125 z^{3}-3900 z^{2}+27060 z+13552+(50 z+220) \sqrt{500 z^{4}-1100 z^{3}-22635 z^{2}+69988 z-395692}, \\
& Q=1125 z^{3}+3900 z^{2}-27060 z-13552+(50 z+220) \sqrt{500 z^{4}-1100 z^{3}-22635 z^{2}+69988 z-395692},
\end{aligned}
$$

as shown in Fig. 1. The overall shape turns out as expected. The membership functions $L(z)$ and $R(z)$ have complex values with their imaginary parts approaching zero when $\frac{17}{62} \leq z \leq \frac{4714}{7945}$ for $L(z)$ and $\frac{112}{115} \leq z \leq \frac{1697}{1175}$ for $R(z)$. Hence, the imaginary parts of these two functions have no influence on the computational results and can be disregarded.

Next, we perform $\alpha$-cuts of batch-arrival and service rates and fuzzy expected number of sewage solids in the system at eleven distinct $\alpha$ values: $0,0.1, \ldots, 1$. Crisp intervals for fuzzy expected number of sewage solids in the system at different possibilistic $\alpha$ levels are presented in Table 1. The fuzzy expected number of sewage solids in the system $\widetilde{N}_{s}$ has two characteristics to be noted. First, the support of $\widetilde{N}_{s}$ ranges from 0.2742 to 1.4443 ; this indicates that, though the expected number of sewage solids in the system is fuzzy, it is impossible for its values to fall below 0.2742 or exceed 1.4443. Second, the $\alpha$-cut at $\alpha=1$ contains the values from 0.5933 to 0.9739 , which are the most possible values for the fuzzy expected number of sewage solids in the system. 


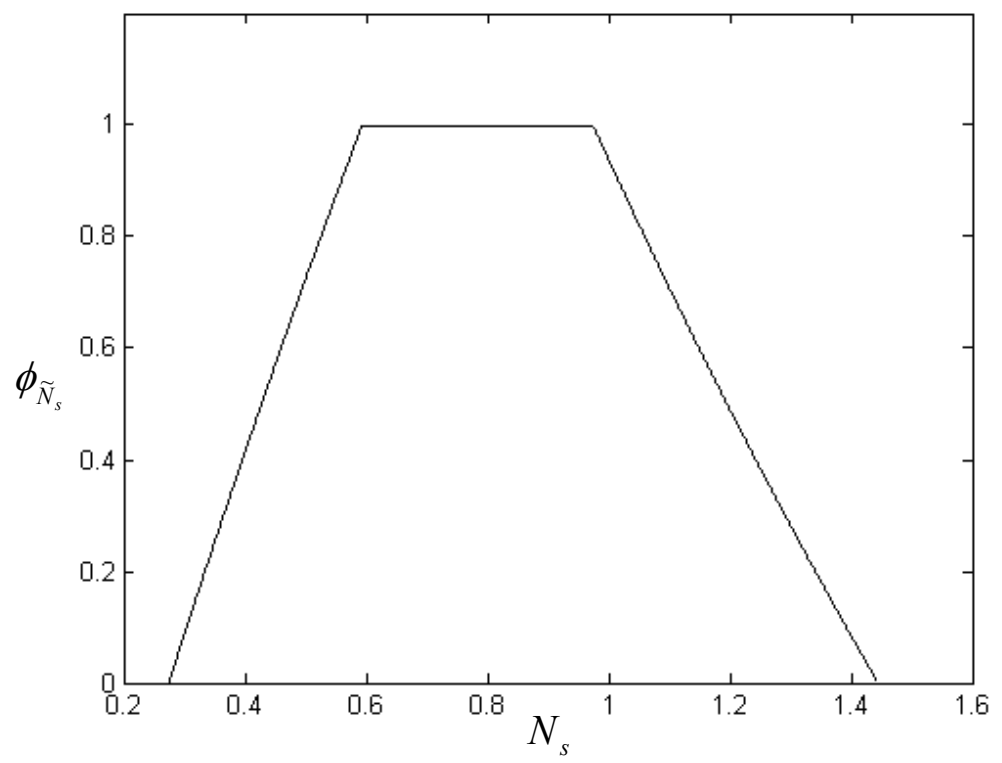

Fig. 1. The membership function for fuzzy expected number of sewage solids in the system

Table 1. $\alpha$-cuts of batch-arrival and service rates and expected number of sewage solids in the system

\begin{tabular}{|c|c|c|c|c|c|c|}
\hline$\alpha$ & $x_{\alpha}^{L}$ & $x_{\alpha}^{U}$ & $y_{\alpha}^{L}$ & $y_{\alpha}^{U}$ & $\left(N_{s}\right)_{\alpha}^{L}$ & $\left(N_{s}\right)_{\alpha}^{U}$ \\
\hline 0.00 & 1.00 & 4.00 & 11.00 & 14.00 & 0.2742 & 1.4443 \\
\hline 0.10 & 1.10 & 3.90 & 11.10 & 13.90 & 0.3039 & 1.3919 \\
\hline 0.20 & 1.20 & 3.80 & 11.20 & 13.80 & 0.3340 & 1.3410 \\
\hline 0.30 & 1.30 & 3.70 & 11.30 & 13.70 & 0.3646 & 1.2912 \\
\hline 0.40 & 1.40 & 3.60 & 11.40 & 13.60 & 0.3957 & 1.2427 \\
\hline 0.50 & 1.50 & 3.50 & 11.50 & 13.50 & 0.4273 & 1.1953 \\
\hline 0.60 & 1.60 & 3.40 & 11.60 & 13.40 & 0.4594 & 1.1491 \\
\hline 0.70 & 1.70 & 3.30 & 11.70 & 13.30 & 0.4920 & 1.1038 \\
\hline 0.80 & 1.80 & 3.20 & 11.80 & 13.20 & 0.5252 & 1.0596 \\
\hline 0.90 & 1.90 & 3.10 & 11.90 & 13.10 & 0.5590 & 1.0163 \\
\hline 1.00 & 2.00 & 3.00 & 12.00 & 13.00 & 0.5933 & 0.9739 \\
\hline
\end{tabular}

Similarly, the membership function for the fuzzy expected waiting time in the queue $\left(\widetilde{W}_{q}\right)$ is obtained as shown in Fig. 2. Crisp intervals for the fuzzy expected waiting time in the queue at different possibilistic $\alpha$ levels are given in Table 2. For the fuzzy expected waiting time $\widetilde{W}_{q}$, the range of $\widetilde{W}_{q}$ at $\alpha=1$ is [0.0714, 0.0790], indicating that expected waiting time for any sewage solids definitely falls between 0.0714 and 0.0790. Moreover, the range of $\widetilde{W}_{q}$ at $\alpha=0$ is $[0.0657,0.0896]$, indicating that the expected waiting time in the queue will never exceed 0.0896 or fall 
below 0.0657 .

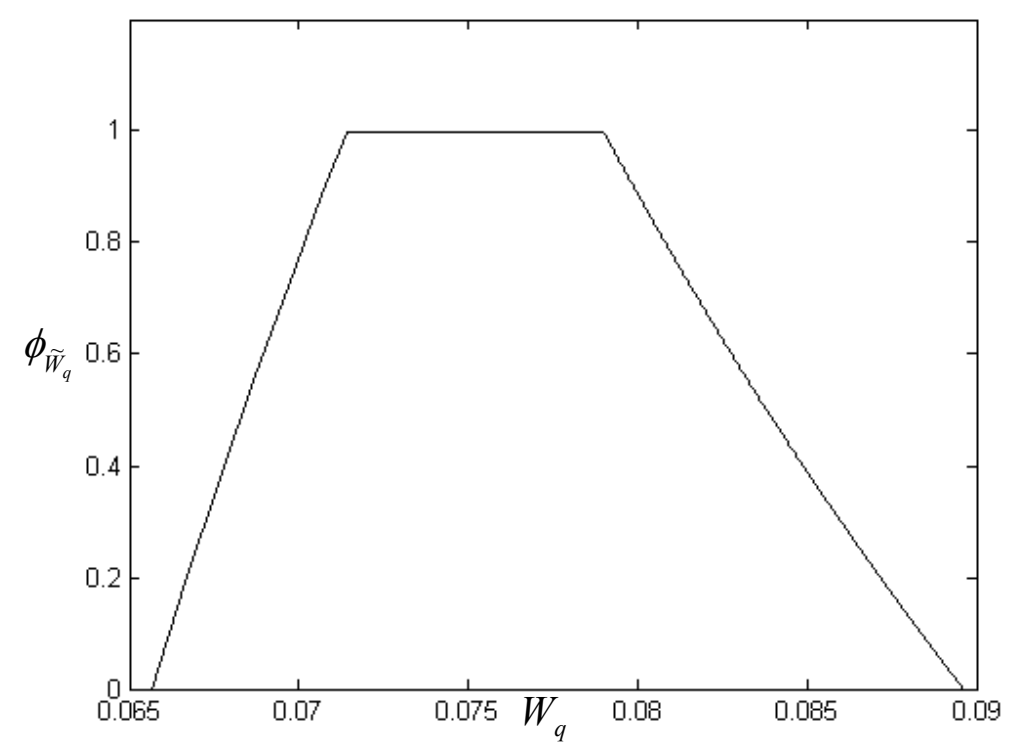

Fig. 2. The membership function for fuzzy expected waiting time in the queue

Table 2. $\alpha$-cuts of batch-arrival and service rates and expected waiting time

\begin{tabular}{|c|c|c|c|c|c|c|}
\hline$\alpha$ & $x_{\alpha}^{L}$ & $x_{\alpha}^{U}$ & $y_{\alpha}^{L}$ & $y_{\alpha}^{U}$ & $\left(W_{q}\right)_{\alpha}^{L}$ & $\left(W_{q}\right)_{\alpha}^{U}$ \\
\hline 0.00 & 1.00 & 4.00 & 11.00 & 14.00 & 0.0657 & 0.0896 \\
\hline 0.10 & 1.10 & 3.90 & 11.10 & 13.90 & 0.0662 & 0.0884 \\
\hline 0.20 & 1.20 & 3.80 & 11.20 & 13.80 & 0.0667 & 0.0872 \\
\hline 0.30 & 1.30 & 3.70 & 11.30 & 13.70 & 0.0672 & 0.0860 \\
\hline 0.40 & 1.40 & 3.60 & 11.40 & 13.60 & 0.0678 & 0.0849 \\
\hline 0.50 & 1.50 & 3.50 & 11.50 & 13.50 & 0.0684 & 0.0838 \\
\hline 0.60 & 1.60 & 3.40 & 11.60 & 13.40 & 0.0689 & 0.0828 \\
\hline 0.70 & 1.70 & 3.30 & 11.70 & 13.30 & 0.0695 & 0.0818 \\
\hline 0.80 & 1.80 & 3.20 & 11.80 & 13.20 & 0.0701 & 0.0808 \\
\hline 0.90 & 1.90 & 3.10 & 11.90 & 13.10 & 0.0708 & 0.0799 \\
\hline 1.00 & 2.00 & 3.00 & 12.00 & 13.00 & 0.0714 & 0.0790 \\
\hline
\end{tabular}

\section{CONCLUSIONS}

This paper applies the concepts of $\alpha$-cuts and Zadeh's extension principle to a batch-arrival queuing system with multiple servers and constructs membership functions of the expected number of customers and the expected waiting time using paired NLP models. Following the proposed approach, $\alpha$-cuts of the membership functions are found and their interval limits inverted to attain explicit closed-form expressions for the system characteristics. Even when the membership function intervals cannot be inverted, system designers or managers can specify the system 
characteristics of interest, perform numerical experiments to examine the corresponding $\alpha$-cuts and then use this information to develop or improve system processes.

For example, in Example, a designer (manager) can set the range of the number of sewage solids to be $[0.5252,1.0596]$ to reflect the desired service and find that the corresponding $\alpha$ level is 0.8 with $y_{\alpha}^{L}=11.80$ and $y_{\alpha}^{U}=13.20$. In other words, the designer can determine that the service rate is between 11.80 and 13.20. Similarly, a designer can also set the expected waiting time with "rounder" numbers like [0.0678, 0.0849 ] to reflect the desired service, and the corresponding $\alpha$ level is 0.4 with $y_{\alpha}^{L}=11.40$ and $y_{\alpha}^{U}=13.60$. As this example demonstrates, the approach proposed in this paper provides practical information for system designers and practitioners.

\section{REFERENCES}

[1] J. Buzacott and J. Shanthikumar, Stochastic Models of Manufacturing Systems, Englewood Cliffs, NJ: Prentice-Hall, 1993.

[2] S. Chanas and M. Nowakowski, Single value simulation of fuzzy variable, Fuzzy Sets and Systems, 21, 43-57, 1988.

[3] X. Chao and Y. Zhao, Analysis of multiple-server queue with station and server vacation, European Journal of Operational Research, 110, 392-406,1998.

[4] S. P. Chen, Parametric nonlinear programming for analyzing fuzzy queues with finite capacity, European Journal of Operational Research, 157, 429-438, 2004.

[5] S. P. Chen, Parametric nonlinear programming approach to fuzzy queues with bulk service. European Journal of Operational Research, 163, 434-444, 2005.

[6] T. Gal, Postoptimal Analysis, Parametric Programming, and Related Topics, New York: McGraw-Hill, 1979.

[7] D. Gross, and C. M. Harris, Fundamentals of Queueing Theory, 3rd Ed, NewYork: JohnWiley, 1998.

[8] N. Igaki, Exponential two server queue with N-policy and multiple vacations, Queueing systems, 10, 279-294,1992.

[9] C. Kao, C. C. Li and S. P. Chen, Parametric programming to the analysis of fuzzy queues, Fuzzy Sets and Systems, 107, 93-100, 1999.

[10] A. Kaufmann, Introduction to the Theory of Fuzzy Subsets, Volume 1, New York: Academic Press, 1975.

[11] L. Kleinrock, Queueing Systems, Vol. I. Theory, New York: Wiley, 1975.

[12] Y. Levy and U. Yechiali, A M/M/c queue with servers vacations, INFOR, 14, 153-163, 1976.

[13] R. J. Li and E. S. Lee, Analysis of fuzzy queues, Computers and Mathematics with Applications, 17, 1143-1147, 1989.

[14] D. S. Negi and E. S. Lee, Analysis and simulation of fuzzy queue, Fuzzy Sets and Systems, 46, 321-330, 1992.

[15] H. M. Prade, An Outline of Fuzzy or Possibilistic Models for Queueing Systems, Fuzzy Sets, Ed. P. P. Wang and S. K. Chang, New York: Plenum Press, (1980)

[16] R.E. Stanford, The set of limiting distributions for a Markov chain with fuzzy transition probabilities, Fuzzy Sets and Systems, 7, 71-78,1982.

[17] N. Tian, Q. Li and J. Cao, Conditional stochastic decompositions in the M/M/c queue with server vacations, Stochastic Models, 14(2), 367-377, 1999. 
[18] N. Tian and $\mathrm{X} . \mathrm{Xu}, \mathrm{M} / \mathrm{M} / \mathrm{c}$ queue with synchronous multiple vacation of partial servers, OR Transactions, 5(3), 85-94, 2001.

[19] K. S. Trivedi, Probability and Statistics with Reliability, Queueing and Computer Science Applications. New York: John Wiley \& Sons Inc, 2002.

[20] B. Vinod, Exponential queue with server vacation, Journal of Operational Research Society, 37, 1007-1014, 1986.

[21] R. R. Yager, A characterization of the extension principle, Fuzzy Sets and Systems, 18, 205-217, 1986.

[22] L. A. Zadeh, Fuzzy sets as a basis for a theory of possibility, Fuzzy Sets and Systems, 1, 3-28, 1978.

[23] G. Zhang and N. Tian, Analysis of queueing systems with synchronous single vacation for some servers, Queueing systems, 45, 161-175, 2003.

[24] G. Zhang and N. Tian, Analysis on queueing systems with synchronous vacations of partial servers, Performance Evaluation, 52, 269-282, 2003.

[25] H. J. Zimmermann, Fuzzy Set Theory and its Applications, 4th Ed, Boston: Kluwer Academic, 2001. 